\title{
3 Riemann-Roch Spaces
}

In this chapter we introduce Riemann-Roch spaces and the classic theorem that connects the dimension of them to the degree of a divisor and to the genus of a function field or curve. We do not give any details on the proof of Riemann-Roch theorem since our target is to understand the structure of the space and to be able to compute it.

The lemmas and propositions that are following can be found widely in literature in similar or the same versions (for example (Stichtenoth, 1993), (Lang, 1983), (Fulton and Lang, 1985), (Jacobson, 1974) and (Atiyah and Macdonald, 1969)). In our case we try to expand where possible and include only those that provide us structure elements.

\subsection{Valuation Rings}

In this chapter we introduce another two very interesting structures of rings called valuation rings and discrete valuation rings. Discrete valuation rings are closely connected to the theory in Chapter 8 for computing rational points on curves.

Definition Let $R$ be an integral domain and $F$ its field of fractions. Then, the set

$$
R=\left\{a \in F \mid a \in R \text { or } a^{-1} \in R\right\}
$$

is called valuation ring.

A very interesting property of valuation rings is that they are local rings, which means that they have exactly one maximal ideal.

Theorem 3.1.1. Every valuation ring is a local ring.

Proof. Let $R$ be a valuation ring and $U_{R}$ be its group of units. We will show that $P=$ $R-U_{R}$, the set of non-units of $R$, is the unique maximal ideal of $R$. Let $p \in P$ and $r \in R$. If $p r \in U_{R}$ this implies that $p$ is a unit which is a contradiction. Now, let $p, q$ be elements of $P$. This implies that either $p / q$ or $q / p$ belongs to $R$. If $q / p$ was an element of $R$ and $p+q$ was a unit it would imply that $p$ is also a unit since

$$
p+y=p(1+y / p) \text {. }
$$

Therefore $p+y$ belongs to $P$. Similarly, if $p / q$ was an element of $R$ and $p+q$ was a unit it would imply that $q$ is a unit. Thus, $P$ is an ideal.

Now suppose that $P$ is not a maximal ideal and $P^{\prime} \supset P$. Since $P$ consists of all nonunits it implies that $P^{\prime}$ contains at least one unit element. Therefore $P^{\prime}=R$. Finally, suppose to obtain a contradiction that $P$ is not the unique maximal ideal and that there is another maximal ideal $P^{\prime \prime}$. Since $P^{\prime \prime}$ is another maximal ideal this means that 
it is not contained in $P^{\prime}$ and therefore it contains unit elements. So, $P$ is the unique maximal ideal of $R$.

For example the set

$$
R=\left\{\frac{a p^{n}}{b} \mid \operatorname{gcd}(p, a)=\operatorname{gcd}(p, b)=1\right\}
$$

is a valuation ring where its field of fractions is the field of rational numbers $\mathbb{Q}$ and its maximal ideal is $(p)$.

Next we will see two of the main properties of valuation rings that connect them to the principal ideal domains, Noetherian domains and integrally closed domains.

Proposition 3.1.2. Every valuation ring $R$ is integrally closed domain in its field of fractions $F$.

Proof. Let $a \in F$ and suppose that it is integral over $R$. If $a \in R$ then $R$ is integrally closed. If $a$ is not in $R$ then $a^{-1}$ belongs to $R$ since $R$ a valuation ring. But, we assumed that $a$ is integral over $R$ and therefore there is a polynomial

$$
a^{n}+r_{n-1} a^{n-1}+\cdots+r_{1} a+r_{0}=0
$$

for some $r_{i} \in R$. By multiplying 3.1.1 by $a^{-n+1}$ we see that

$$
a+r_{n-1}+r_{n-2} a^{-1}+\cdots+r_{1} a^{-n+2}+r_{0} a^{-n+1}=0
$$

which implies that

$$
a=-r_{n-1}-r_{n-2} a^{-1}-\cdots-r_{1} a^{-n+2}-r_{0} a^{-n+1}=0 .
$$

Since we assumed that $a^{-1} \in R$ it implies that $a \in R$, and therefore $R$ is integrally closed.

Proposition 3.1.3. The ideals of a valuation ring $R$ are totally ordered.

Proof. Let $I, J$ be two ideals of $R$ and suppose without loss of generality that $I$ is not a subideal of $J$. Let $a \in I-J$ and $b \in J$. If $a / b$ belongs to $R$ then

$$
a=(a / b) b \Rightarrow a \in J
$$

which is a contradiction. Therefore, since $R$ is a valuation ring $(a / b)^{-1}=(b / a)$ belongs to $R$ which means that

$$
b=(b / a) a \in R \Rightarrow b \in I
$$

Hence, $J \subset I$.

Proposition 3.1.4. A valuation ring $R$ is a principal ideal domain if and only if it is Noetherian Domain. 
Proof. Suppose that $R$ is Noetherian. By definition every ideal of $R$ is finitely generated and by 3.1.3 we have that the ideals of $R$ are totally ordered. Thus, if $I=\left(a_{1}, \ldots, a_{n}\right)$, by 3.1 .3 without loss of generality we get that

$$
\left(a_{1}\right) \subset\left(a_{2}\right) \subset \cdots \subset\left(a_{n}\right)
$$

and therefore $I=\left(a_{n}\right)$ is a principal ideal.

The converse is obvious since every principal ideal domain is a Noetherian domain (proposition 1.1.10).

Now let $R$ be an integral domain. We define the element $\infty$ with the following properties

- $a \pm \infty=\infty, \forall a \in R$.

- $a \cdot \infty=\infty, \forall a \in R-\{0\}$.

- $\infty \cdot \infty=\infty$.

- $\frac{1}{\infty}=0$.

- $\frac{1}{0}=\infty$.

Definition Let $F / K(x)$ be a function field. The surjective map

$$
v: F \rightarrow \mathbb{Z} \cup\{\infty\}
$$

that satisfies the following three conditions:

- $v(a b)=v(a)+v(b)$,

- $v(a+b) \geq \min \{v(a), v(b)\}$,

- $v(0)=\infty$

is called discrete valuation.

An immediate consequence of the definition of the discrete valuation is the following lemma.

Lemma 3.1.5. Let $v$ be a discrete valuation and $a, b \in F / K(x)$ such that $v(a) \neq v(b)$, then

$$
v(a+b)=\min \{v(a), v(b)\}
$$

Proof. Since $v(a) \neq v(b)$ we can assume without loss of generality that $v(a)<v(b)$. Assume now that $v(a+b) \neq \min \{v(a), v(b)\}$. Thus, we have that $v(a+b)>v(a)$ which implies

$$
v(a)=v((a+b)-b) \geq \min \{v(a+b), v(-b)\} .
$$

Hence

$$
v(a) \geq \min \{v(a+b), v(b)\}>\min \{v(a), v(b)\}>v(a)
$$

which is a contradiction. Thus $v(a+b)=\min \{v(a), v(b)\}$. 
The following proposition connects the valuation rings with the discrete valuation rings.

Proposition 3.1.6. Let $v$ be a discrete valuation on the function field $F / K(x)$, then

(i) $V=\{a \in F \mid v(a) \geq 0\}$ is a valuation ring.

(ii) $U_{V}=\{a \in F \mid v(a)=0\}$ is the unit group of $V$.

(iii) $P=\{a \in F \mid v(a)>0\}$ is the maximal ideal of $V$,

Proof. (i). By the definition of the valuation ring it is easy to see that $V$ is a ring. In order $V$ to be a valuation ring, we need to show that for every element $a$ of $F, a \in V$ or $a^{-1} \in V$. Let $a \in F$ such that $a \notin V$. Thus, $v(a)<0$. Now, since $a \cdot a^{-1}=1$ we have that

$$
v\left(a \cdot a^{-1}\right)=v(1) \Rightarrow v(a)+v\left(a^{-1}\right)=v(1) .
$$

For $a=1$ we get that $v(1)=0$ and therefore $v\left(a^{-1}\right)=-v(a)>0$. So $a^{-1} \in V$, which implies that $V$ is a valuation ring.

(ii). If $a$ is a unit of $V$ it implies that both $a$ and $a^{-1}$ are elements of $V$. In that case both $v(a)$ and $v\left(a^{-1}\right)$ are greater or equal to zero. This can only happened if and only if $v(a)=v\left(a^{-1}\right)=0$.

(iii). The maximal ideal of $V$ consists of all nonunits of $V$ (proposition 1.2.1). The result follows from (i) and (ii).

Proposition 3.1.6 leads us to the definition of the discrete valuation ring.

Definition If $v$ is a discrete valuation on a function field $F / K(x)$, then the set

$$
V=\{a \in F \mid v(a) \geq 0\}
$$

is called a discrete valuation ring.

Obviously, all discrete valuation rings are valuation rings. The converse doesn't always hold.

Definition Let $V$ be a discrete valuation ring defined by the discrete valuation $v$ and $P$ its maximal ideal. An element $t$ is called uniformizer or prime element for $P$ if and only if $v(t)=1$.

Proposition 3.1.7. Let $F / K(x)$ be a function field, $V$ a discrete valuation ring and the uniformizer of the maximal ideal $P$ of $V$. It holds that

(i) $t$ generates $P$,

(ii) every nonzero element a of $F$ has a unique representation of the form $a=u t^{n}$ where $u$ is a unit of $V$ and $n$ an integer.

(iii) $V$ is a principal ideal domain and for all ideals I of $V$ there is $t \in V$ such that $I=\left(t^{n}\right)$. 
Proof. (i). Let $(t)$ be the principal ideal generated by the uniformizer $t$. Since $P$ is maximal and unique we have that $(t) \subset P$. Let $p$ be an element of $P$. According to 3.1.6, $v(p)>0$ where $v$ is the discrete valuation associated to the discrete valuation ring $V$. On the other hand, we have

$$
v\left(p t^{-1}\right)=v(p)+v\left(t^{-1}\right)=v(p)-v(t)=v(p)-1 \geq 0 .
$$

So, $p t^{-1}$ belongs to $V$ and since $t^{-1} \notin V$ it implies that $p \in(t)$. Therefore $P=(t)$.

(ii). Let $v$ be the discrete valuation, such that $v(a)=n$. From $a=u t^{n}$ we get that $u=a t^{-n}$. We can easily see that

$$
v\left(a t^{-n}\right)=v(a)+v\left(t^{-n}\right)=v(a)+v\left(t^{n}\right)=n-n=0
$$

which implies by proposition 3.1.6 that $u$ is a unit. This establishes the uniqueness of the representation.

(iii). Let $I$ be a non-zero subideal of the maximal ideal $P$ and $a \in I$ such that $v(a)=n$ to be the smallest. By (ii) we have that there is $u, t \in V$ such that $a=u t^{n}$ which implies that $t^{n}=a u^{-1}$. Since $P$ is generated by $t$ it implies that $\left(t^{n}\right) \subset I$.

Conversely, let $a^{\prime}$ be an element of $I$ with $v\left(a^{\prime}\right)=m$. Thus

$$
v\left(a^{\prime} t^{-m}\right)=v\left(a^{\prime}\right)+v\left(t^{-m}\right)=v\left(a^{\prime}\right)+v\left(t^{m}\right)=m-m=0
$$

and therefore $a^{\prime} t^{-m}$ is a unit. Hence there is $u^{\prime}$ such that $a^{\prime}=u^{\prime} t^{m}$ and since $m \geq n$ therefore $a^{\prime} \in\left(t^{n}\right)$. So $I \subset\left(t^{n}\right)$.

Discrete valuation rings have actually more and very interesting and useful properties. For example every discrete valuation rings since they are principal ideal domains it follows that they are Dedekind domains and unique factorizations domains with actually a unique irreducible element.

\subsection{Places and Divisors}

In this section we will deal with the discrete valuation rings of a function field and especially with their maximal ideals.

Definition The maximal ideals of the discrete valuation rings of a function field are called places.

Since the maximal ideal $P$ of a discrete valuation ring $V$ is unique we can determine the discrete valuation ring by its place. We will denote the discrete valuation ring $V$ with maximal ideal $P$ by $V_{P}$. The set of the places of a function field $F / K(x)$ will be denoted by $\mathbb{P}_{F}$ and we will see that it is an infinite set. 
Definition Let $F / K(x)$ be a function field, $a$ an element of it and $P$ a place in $\mathbb{P}_{F}$. We say that $P$ is a zero of $a$ if and only if $v(a)>0$ and is a pole of $a$ if and only if $v(a)<0$, where $v$ is the discrete valuation associated to the place $P$.

It is proved (Stichtenoth, 1993) that the poles and and zeros of an element of a function field are finite.

Since a place is a maximal ideal $V_{P} / P$ will always be a field and this allow us to define the degree of $P$.

Definition Let $F / K(x)$ be a function field and $P \in \mathbb{P}_{F}$. The field $F_{P}=V_{P} / P$ is called residue class field of $P$. The dimension of $F_{P}$ as a $K$-vector space is called the degree of the place $P$.

In order to understand better the structure of a place of a function field we will try initially to identify the places of a rational function field $K(x) / K$. For every irreducible polynomial $p(x) \in K(x)$ we define the discrete valuation rings

$$
V_{p(x)}=\left\{\frac{f(x)}{g(x)} \mid f(x), g(x) \in K[x], p(x) \vee g(x)\right\}
$$

and their places

$$
P_{p(x)}=\left\{\frac{f(x)}{g(x)}|f(x), g(x) \in K[x], p(x)| f(x), p(x) \vee g(x)\right\} .
$$

Besides $V_{p(x)}$, another valuation ring of the function field is

$$
V_{\infty}=\left\{\frac{f(x)}{g(x)} \mid f(x), g(x) \in K[x], \operatorname{deg} f(x) \leq \operatorname{deg} g(x)\right\}
$$

and its place is:

$$
P_{\infty}=\left\{\frac{f(x)}{g(x)} \mid f(x), g(x) \in K[x], \operatorname{deg} f(x)<\operatorname{deg} g(x)\right\} .
$$

$P_{\infty}$ is called place at infinity and together with the places $P_{p(x)}$, they form the set $\mathbb{P}_{K(x)}$.

Definition Let $F^{\prime} / K^{\prime}(x)$ be a function field extension of $F / K(x)$. We say that a place $P^{\prime}$ of $\mathbb{P}_{F^{\prime}}$ lies over or is an extension of $P$ if and only if $P \subset P^{\prime}$.

Since all algebraic fields $F / K(x)$ are extensions of the rational function field $K(x) / K$, this implies that all places $\mathbb{P}_{F}$ are lying over (are extensions) of a place of $\mathbb{P}_{K(x)}$. The set of places lying over the place at infinity $P_{\infty}$ is called the set of infinite places. The set of places form a free $\mathbb{Z}$-module. This leads us to the next definition.

Definition We call divisor of a function field the sum

$$
D=\sum_{\mathbb{P}_{F}} n_{P} P
$$


where all but finite $n_{P} \in \mathbb{Z}$ are equal to zero. We denote the group of divisors by $\mathbb{D}_{F}$.

By setting $v_{P}(D)=n_{P}$ we can define an ordering relation as follows. Let $D_{1}$ and $D_{2}$ be divisors of $\mathbb{D}_{F}$, then

$$
D_{1} \leq D_{2} \Longleftrightarrow v_{P}\left(D_{1}\right) \leq v_{P}\left(D_{2}\right)
$$

for all $P \in \mathbb{P}_{F} . D_{1}$ is called subdivisor of $D_{2}$ if and only if $D_{1} \leq D_{2}$.

Now let $a \in F / K(x)$ be a non-zero element with $P_{1}, \ldots, P_{m}$ zeros and $Q_{1}, \ldots, Q_{n}$ poles. We define

$$
(x)=(x)_{0}-(x)_{\infty}
$$

to be the principal divisor of $a$, where

$$
(x)_{0}=\sum_{i=1}^{m} v_{P_{i}} P_{i}
$$

is the zero divisor of $a$ and

$$
(x)_{\infty}=-\sum_{i=1}^{n} v_{Q_{i}} Q_{i}
$$

is the pole divisor of $a$. Two divisors $D_{1}$ and $D_{2}$ are called equivalent if

$$
D_{1}=D_{2}+(y)
$$

for some $y \in F$.

Definition Let $F / K(x)$ be a function field and

$$
D=\sum_{i=1}^{m} n_{P_{i}} P_{i}
$$

a divisor of $\mathbb{D}_{F}$. The sum

$$
\operatorname{deg} D=\sum_{i=1}^{m} v_{P_{i}}(D) \operatorname{deg} P_{i}
$$

is called the degree of $D$.

Finally, we present a corollary of Kummer's theorem as it is proved in (Stichtenoth, 1993). Kummer's corollary provides us a relation between the places of an arbitrary function field $F / K(x)$ and the rational function field $K(x) / K$. We denote by $P_{a}$ the place of $\mathbb{P}_{K}(x)$ that is equal to $P_{p(x)}$ where $p(x)=x-a$.

Corollary 3.2.1 (Kummer's Corollary). Let

$$
f(t)=t^{n}+f_{n-1}(x) t^{n-1}+\cdots+f_{1}(x) t+f_{0}(x)
$$


be a polynomial of $K(x)[t]$ irreducible over $K(x)$. Let $y$ such that $f(y)=0$ and $a \in K$ such that $f_{j}(a) \neq \infty$ for all $j \in\{0, \ldots, n-1\}$. Set

$$
f_{a}(t)=t^{n}+f_{n-1}(a) t^{n-1}+\cdots+f_{1}(a) t+f_{0}(a) .
$$

Suppose that

$$
f_{a}(t)=\prod_{i=1}^{r} \phi_{i}(t),
$$

where $\phi_{i}(t)$ irreducible, monic, pairwise distinct polynomials of $K[t]$. It holds that

(i) for every $i \in\{1, \ldots, r\}$ there is a unique place $P_{i} \in \mathbb{P}_{K(x, y)}$ such that $x-a \in P_{i}$ and $\phi_{i}(y) \in P_{i}$.

(ii) if $f_{a}(t)$ has $n$ distinct roots in $K$, then for every $b$ for which $f_{a}(b)=0$ there is a place $P_{a, b} \in \mathbb{P}_{K}(x, y)$ such that $x-a$ and $y-b$ are elements of $P_{a, b}$. Moreover, the degree of $P_{a, b}$ is equal to 1 .

Kummer's initial theorem can be found also in (Stichtenoth, 1993) and in (Cassels and Fröhlich, 1967).

\subsection{Riemann-Roch Space}

Definition Let $F / K(x)$ be a function field and $D$ a divisor of $\mathbb{D}_{F}$. The set

$$
L(D)=\{a \in F / K(x) \mid a \neq 0,(a) \geq-D\} \cup\{0\}
$$

is called Riemann-Roch space of $D$.

The following lemma justifies why $L(D)$ is called a space.

Lemma 3.3.1. Let $F / K(x)$ be a function field. For any divisor $D$ the Riemann-Roch space $L(D)$ is a K-vector space.

Proof. Let $a, b \in L(D)$ and $k \in K$. For every place of $\mathbb{P}_{F}$ we have that

$$
v_{P}(a+b) \geq \min \left\{v_{P}(a), v_{P}(b)\right\} \geq-v_{P}(D)
$$

and

$$
v_{P}(k a)=v_{P}(k)+v_{P}(a) \geq-v_{P}(D) .
$$

Thus, $a+b$ and $k a$ belong to $L(D)$ and therefore $L(D)$ is a $K$-vector space.

Suppose that

$$
D=\sum_{i=1}^{r} n_{i} P_{i}-\sum_{j=1}^{s} m_{j} Q_{j}
$$


where $P_{i}$ are the zeros, $Q_{j}$ are the poles and $n_{i}, m_{j} \in \mathbb{N}$. From the relation $(a) \geq-D$ we understand that $L(D)$ consists of all functions of the function field that have zeros of orders greater or equal to $m_{1}, \ldots, m_{s}$ in the points $Q_{1}, \ldots, Q_{s}$, respectively, and poles at the points $P_{1}, \ldots, P_{r}$ of orders at most $n_{1}, \ldots, n_{r}$, respectively.

Lemma 3.3.2. Let $F / K(x)$ be a function field and $D, D_{1}, D_{2} \in \mathbb{D}_{F}$. We have that

(i) $a \in L(D)$ if and only if $v_{P}(a) \geq-v_{P}(D)$ for all $P \in \mathbb{P}_{F}$.

(ii) If $D_{1} \leq D_{2}$, then $L\left(D_{1}\right) \subset L\left(D_{2}\right)$.

(iii) $L\left(D_{1}\right) \neq\{0\}$ if and only if there is a divisor $D_{2}$ equivalent to $D_{1}$ such that $D_{2} \geq 0$.

Proof. (i) Let

$$
\text { (a) }=\sum_{i=1}^{r} n_{i} P_{i}-\sum_{j=1}^{s} m_{j} Q_{j} .
$$

Obviously, $a \in L(D)$ if and only if $(a) \geq-D$, which holds if and only if $v_{p}(a) \geq-v_{P}(D)$.

(ii) Since $D_{1} \leq D_{2}$, it implies that $v_{P}\left(D_{1}\right) \leq v_{P}\left(D_{2}\right)$ for all $P \in \mathbb{P}_{F}$. If $a \in L\left(D_{1}\right)$, then

$$
v_{P}(a) \geq-v_{P}\left(D_{1}\right) \geq-v_{P}\left(D_{2}\right) .
$$

Thus, $a \in L\left(D_{2}\right)$.

(iii) $L\left(D_{1}\right)$ is not the trivial field if and only if there is a non-zero $a \in F$ such that

$$
(a) \geq-D_{1} \Longleftrightarrow(a)+D_{1} \geq 0 .
$$

By setting $D_{2}=(a)+D_{1}$ the result follows.

Definition The map

$$
\omega: \mathbb{P}_{F} \rightarrow F,
$$

where $\omega(P) \in F$ for all but finitely many $P$ of $\mathbb{P}_{F}$, is called adele of $F / K(x)$. The set of all adeles $\Omega_{F}$ form a $K$-vector space that is called adele space. For a divisor $D \in \mathbb{D}_{F}$ we define the set

$$
\Omega_{F}(D)=\left\{\omega \in \Omega_{F} \mid v_{p}(\omega)+v_{p}(D) \geq 0, \forall P \in \mathbb{P}_{F}\right\}
$$

to be the adele subspace of $D$.

The dimension as a $K$-vector space of the quotient field

$$
\Omega_{F} /\left(\Omega_{F}(D)+F\right)
$$

it called index speciality of $D$ and is denoted by $i(D)$. Index of speciality is actually an invariant for function fields.

Another invariant of the function fields is their genus. We define as genus of a function field defined by a polynomial

$$
f(x, y)=a_{n}(x) y^{n}+\cdots a_{1}(x) y+a_{0}(x)
$$

where $a_{i}(x)$ are elements of $K(x)$ the genus of the non-singular model of the curve $\mathrm{C}$ defined by $f(x, y)$. 
Theorem 3.3.3 (Riemann-Roch Theorem). Let $g$ be the genus of the function field $F / K(x)$. It holds that

$$
\operatorname{dim} D-\operatorname{deg} D=i(D)-g+1
$$

for every $D \in \mathbb{D}_{F}$.

The proof of this well known theorem can be found in several textbooks of the literature such as (Stichtenoth, 1993), (Fulton and Lang, 1985) and (Fried and Jarden, 1986).

\subsection{Holomorphy Rings}

In this last section of the chapter we introduce another one algebraic structure with a lot of interesting properties.

Definition Let $F / K(x)$ be a function field and $\Sigma$ a set of places. The ring

$$
V_{\Sigma}=\left\{a \in F \mid v_{P}(a) \geq 0, \forall P \in \Sigma\right\}
$$

is called holomorphy ring and the ring

$$
V^{\Sigma}=\left\{a \in F \mid v_{P}(a) \geq 0, \forall P \notin \Sigma\right\}
$$

is called complement of the holomorphy ring $V_{\Sigma}$.

If $\Sigma=\{P\}$ has a unique element then it is the discrete valuation ring $V_{P}$. Therefore, a holomorphy ring is the intersection of all $V_{P}$ where $P \in \Sigma$. Obviously, every holomorphy ring has a unique complement which it is also a holomorphy ring.

Proposition 3.4.1. Let $V_{\Sigma}$ be a holomorphy ring of the function field $F / K(x)$.

(i) $V_{\Sigma}$ is integrally closed.

(ii) The field of fractions of $V_{\Sigma}$ is $F$.

(iii) $V_{\Sigma}$ is a principal ideal domain.

(iv) The integral closure $\hat{R}$ of a ring $R$ in $F$ is equal to $V_{\Sigma}$ where $\Sigma$ is the set of all places that $R \subset V_{P}$.

(v) The cardinality of an integral basis of $V_{\Sigma}$ over $R$ is equal to $[F: K(x)]$.

Proof. Detail proof of all of the above properties of the holomorphy rings can be found in (Stichtenoth, 1993).

By the above proposition for $R=V_{\infty}$ we get that $\hat{V}_{\infty}$ is equal to $V_{\Sigma}$ where $\Sigma$ is the set of infinite places of the function field and $\hat{V}_{\infty}$ has integral basis over $V_{\infty}$ of $[F: K(x)]$ elements. Obviously, the complementary holomorphy ring of $V_{\Sigma}$ is $\left.V^{\Sigma}=K \hat{[} x\right]$ and its also a ring with basis of $n$ elements. 
We can represent a divisor $D$ as follows

$$
D=\sum_{i=1}^{r} n_{i} P_{i}-\sum_{j=1}^{s} m_{j} Q_{j}
$$

where, $n_{i}, m_{j} \in \mathbb{Z}, Q_{i}$ are infinite places of $\Sigma$ and $P_{i}$ are the places that are not in $\Sigma$. For convenience we call this representation the representation on infinite places. The next proposition due to Hess provides us another way to represent a divisor.

Lemma 3.4.2. Let $F / K(x)$ be a function field and $\Sigma$ the set of infinite places. Then there is a bijection between the places of $F / K(x)$ and the prime ideals of $V^{\Sigma}$ and $V_{\Sigma}$.

Proof. Since by 3.4.1 the holomorphy rings $V^{\Sigma}$ and $V_{\Sigma}$ are principal ideal domains it implies that the prime ideals are maximal ideals. The maximal ideals of $V^{\Sigma}$ and $V_{\Sigma}$ are $V^{\Sigma} \cap P$ for all $P \notin \Sigma$ and $V_{\Sigma} \cap Q$ for all $Q \in \Sigma$. So the map

$$
\begin{aligned}
\phi: \mathbb{P}_{F} & \rightarrow V(P) \\
P & \mapsto V^{\Sigma} \cap P, \quad P \notin \Sigma \\
Q & \mapsto V_{\Sigma} \cap Q, \quad Q \in \Sigma
\end{aligned}
$$

is a bijection.

Proposition 3.4.3. Let $F / K(x)$ be a function field and $\Sigma$ the set of infinite places. If $\mathrm{J}^{\Sigma}$ and $\mathcal{J}_{\Sigma}$ are the sets of ideals of $V^{\Sigma}$ and $V_{\Sigma}$, respectively, then there is a bijection between the sets $\mathbb{D}_{F}$ and $\mathcal{J}^{\Sigma} \times \mathcal{J}_{\Sigma}$.

Proof. Let

$$
D=\sum_{i=1}^{r} n_{i} P_{i}-\sum_{j=1}^{s} m_{j} Q_{j}
$$

be the representation of $D$ on infinite places. According to the previous lemma there is a bijection between the places of $F / K(x)$ and the prime ideals of $V^{\Sigma}$ and $V_{\Sigma}$. Thus, the map

$$
\begin{aligned}
\psi: \mathbb{D}_{F} & \rightarrow \mathcal{J}^{\Sigma} \times \mathcal{J}_{\Sigma} \\
D & \mapsto\left(\sum_{i=1}^{r} n_{i}\left(P_{i} \cap V^{\Sigma}\right)-\sum_{j=1}^{s} m_{j}\left(Q_{j} \cap V_{\Sigma}\right)\right)
\end{aligned}
$$

is a bijection.

Hence, by the last proposition we deduce that we can represent every divisor of $\mathbb{D}_{F}$ uniquely with the ordered couple $\left(D^{\Sigma}, D_{\Sigma}\right)$ where

$$
\left(D^{\Sigma}, D_{\Sigma}\right)=\left(\prod_{i=1}^{r}\left(P_{i} \cap V^{\Sigma}\right)^{n_{i}}, \prod_{j=1}^{s}\left(Q_{i} \cap V_{\Sigma}\right)^{m_{i}}\right) .
$$


This representation of the divisor $D$ is called ideal representation and it will help us to compute the Riemann-Roch spaces. We recall that if $I$ is an ideal of an integral domain $V$ where $F$ is its fields of fractions, the set

$$
I^{-1}=\{a \in F \mid a I \subset V\}
$$

is called inverse ideal of $I$.

Theorem 3.4.4 (Hess' Theorem). Let $F / K(x)$ be a function field and

$$
D=\sum_{i=1}^{r} n_{i} P_{i}-\sum_{j=1}^{s} m_{j} Q_{j} .
$$

the representation of $D$ on infinite places. It holds that

$$
L(D)=\left(D^{\Sigma}\right)^{-1} \cap\left(D_{\Sigma}\right)^{-1}
$$

where $\left(D^{\Sigma}, D_{\Sigma}\right)$ is the ideal representation of $D$.

Proof. By definition of the inverse ideal, the elements that belong to $\left(D_{\Sigma}\right)^{-1}$ are exactly the $u$ for which $u \cdot D_{\Sigma} \subset V_{\Sigma}$. In $D_{\Sigma}$ belong all functions that have at $Q_{j}$ order at least $m_{j}$. Thus, in $\left(D_{\Sigma}\right)^{-1}$ belong the functions that at $Q_{j}$ have order at most $m_{j}$. Analogously, the elements that belong to $\left(D^{\Sigma}\right)^{-1}$ are all functions that have at $P_{i}$ order at least $n_{i}$. The intersection of those two inverse ideals gives us the function field $L(D)$.

The following corollary is an immediate consequence of the Hess' theorem and is needed in order to compute Riemann-Roch spaces by Hess' algorithm.

Corollary 3.4.5. Let $D$ be a divisor of $\mathbb{D}_{F}$ and $\left(D^{\Sigma}, D_{\Sigma}\right)$ its ideal representation. Then the ideal representation of $D+r \cdot(x)_{0}$, where $r \in \mathbb{Z}$, is $\left(x^{r} \cdot D^{\Sigma}, D_{\Sigma}\right)$ and the ideal representation of $D+r \cdot(x)_{\infty}$ is $\left(D^{\Sigma}, x^{-r} \cdot D_{\Sigma}\right)$. 